\title{
Dynamical mechanism of anticipating synchronization in excitable systems
}

\author{
Marzena Ciszak, ${ }^{1}$ Francesco Marino, ${ }^{2}$ Raúl Toral, ${ }^{1,2}$ and Salvador Balle ${ }^{1,2}$ \\ ${ }^{1}$ Departament de Física, Universitat de les Illes Balears \\ ${ }^{2}$ Instituto Mediterráneo de Estudios Avanzados (IMEDEA), CSIC-UIB, \\ Ed. Mateu Orfila, Campus UIB, 07122 Palma de Mallorca, Spain
}

(Dated: October 29, 2018)

\begin{abstract}
We analyze the phenomenon of anticipating synchronization of two excitable systems with unidirectional delayed coupling which are subject to the same external forcing. We demonstrate for different paradigms of excitable system that, due to the coupling, the excitability threshold for the slave system is always lower than that for the master. As a consequence the two systems respond to a common external forcing with different response times. This allows to explain in a simple way the mechanism behind the phenomenon of anticipating synchronization.
\end{abstract}

The synchronization of nonlinear dynamical systems is a phenomenon common to many fields of science ranging from biology to physics [1], and it has been an active research subject since the work by Huygens in 1665. Recently, the synchronization of chaotic systems in a unidirectional coupling configuration has attracted a great interest due to its potential applications to secure communication systems [2]. Particular attention has been payed to the so-called anticipating synchronization regime, an idea first proposed by Voss in [3]. He showed that, in some parameter regions, two identical chaotic systems can be synchronized by unidirectional delayed coupling in such a manner that the "slave" (the system with coupling) anticipates the "master" (the one without coupling). More specifically, the coupling scheme proposed in [3] for the dynamics of the master, $x(t)$, and slave, $y(t)$ is the following:

$$
\begin{aligned}
& \dot{x}=F(x) \\
& \dot{y}=F(y)+K\left(x-y^{\tau}\right)
\end{aligned}
$$

where $y^{\tau} \equiv y(t-\tau)$. For appropriate values of the delay time $\tau$ and coupling strength $K$, the basic result is that $y(t) \approx$ $x(t+\tau)$, i.e. the slave "anticipates" by an amount $\tau$ the output of the master.

This regime and its stability has been theoretically studied in several systems, from the simplest ones described by linear differential equations and maps where the mathematical details can be fully worked out[4, 5], to the more complicated ones such as semiconductor lasers [6] operating in the chaotic regime. Experimental evidence of anticipating synchronization has been shown in Chua circuits [7] and in semiconductor lasers with optical feedback [8].

This same phenomenon has recently been shown to occur also when the dynamics, instead of chaotic, is excitable. In refs. [9] the effects of unidirectional delayed coupling between two identical excitable systems was studied for both the FitzHugh-Nagumo [10] and Hodgkin-Huxley [11] models. It was shown that, when both systems are excited by the same noise, and for a certain range of coupling parameters, the randomly distributed pulses of the master are preceded by those of the slave. This allows for predicting the occurrence of excitable pulses in the master. Since many biological systems (as neurons and heart cells) exhibit excitable behavior and they often operate in feedback regime in a noisy environment, the study of the delayed coupling effects in a presence of noise is certainly of wide concern.

The anticipating synchronization regime has been often described as a rather counterintuitive phenomenon because of the possibility of the slave system anticipating the unpredictable evolution of the master [3, 5, 7]. The aim of this paper is to provide a simple clear physical mechanism for this regime in delayed coupled excitable systems, showing that the anticipation of the slave is due to a reduction of its excitability threshold induced by the delayed coupling term. As a consequence, the master and the slave respond to a common external forcing with different response times. The proposed dynamical picture allows us to explain all the general features of the phenomenon as well as to determine in a natural way the maximum permitted anticipation time. The results are sustained by numerical integration of the dynamical equations as well as by simple analytical calculations.

A dynamical system commonly used to study excitable behavior is Adler's equation, [12]

$$
\dot{x}=\mu-\cos x
$$

where $x$ is an angular variable (modulo $2 \pi$ ) and $\mu$ the control parameter. For $|\mu|<1$, there are two fixed points at $x_{ \pm}= \pm \arccos \mu$, one being a stable focus $\left(x_{-}\right)$and the other $\left(x_{+}\right)$an unstable saddle point. If $|\mu|>1$, there are no fixed points, and the flow consists in an oscillation of the variable $x$. This limit cycle develops through an Andronov bifurcation at $\mu_{c}= \pm 1$ [13, 14], where the two fixed points collide and annihilate. For $|\mu|<1$, the system displays excitable behavior: if we kick the system out of its stable state with a large enough perturbation, the trajectory will return to the initial state (modulo $2 \pi$ ) through an orbit that closely follows the heteroclinic connection of the saddle and the node. During this orbit, the system is barely sensitive to external perturbations of moderate amplitude.

In order to study anticipating synchronization, we consider two identical Adler's systems with delayed unidirectional coupling under the effect of an external perturbation $I(t)$ acting 


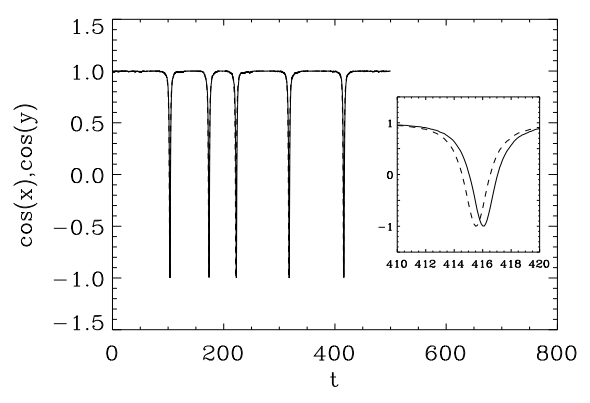

FIG. 1: Time series of the master system $x$ (solid line) and slave system $y$ (dashed line) subjected to white Gaussian noise of zero mean and correlations $\left\langle\left(\xi(t) \xi\left(t^{\prime}\right)\right\rangle=D \delta\left(t-t^{\prime}\right)\right.$, obtained by numerical simulation of eqs. 45. Other parameters are: $\mu=0.95, K=0.01$ $\tau=1$. The noise intensity is $D=0.017$.

simultaneously on both systems,

$$
\begin{aligned}
& \dot{x}=\mu-\cos (x)+I(t) \\
& \dot{y}=\mu-\cos (y)+K\left(x-y^{\tau}\right)+I(t)
\end{aligned}
$$

When $I(t)=\xi(t)$ is zero-mean Gaussian noise, anticipating synchronization occurs as shown in Fig. 11 where we plot the master and slave outputs for a particular value of $K$ and $\tau$. Note that the slave system anticipates the firing of a pulse in the master by a time interval approximately equal to $\tau$. If we increase the coupling constant $K$ or the delay time $\tau$ beyond some values, anticipating synchronization is degraded, i.e., the slave system can emit pulses which do not have a corresponding pulse in the master's output, although the reverse case never occurs. Upon further increasing $K$ or $\tau$, the anticipation phenomenon disappears. The results are analogous to those obtained in [9] for the FitzHugh-Nagumo model.

In order to understand the mechanism of the observed phenomenon, we analyze the behavior of the master alone under the effect of a single perturbation $I(t)=p_{0} \delta\left(t-t_{0}\right)$ acting at a certain time $t_{0}$. The effect of this perturbation appears only as a discontinuity of the, say, $x(t)$ variable at time $t_{0}$ as $x\left(t_{0}^{+}\right)=x\left(t_{0}^{-}\right)+p_{0}$. The condition for the perturbation to be larger than the excitability threshold, is that $x\left(t_{0}^{+}\right)>x_{+}$. ¿From now on, we set the initial condition to be in the rest state, $x\left(t_{0}^{-}\right)=x_{-}$, such the minimum value for the amplitude in order to excite a pulse is $p_{0}>2 \arccos \mu$ and the system develops a pulse after a certain response time $t_{r}$. This time can be precisely defined as the time it takes $x(t)$ to reach a given reference value, e.g. $x_{r}=\pi / 2$. From Eq. (2) we have $t_{r}=\int_{x\left(t_{0}^{+}\right)}^{\pi / 2} \frac{d x}{\mu-\cos x}$ which yields

$$
t_{r}=\frac{1}{\sqrt{1-\mu^{2}}} \ln \left[\frac{(1-b)\left(1+b^{-1} \tan \frac{x\left(t_{0}^{+}\right)}{2}\right)}{(1+b)\left(1-b^{-1} \tan \frac{x\left(t_{0}^{+}\right)}{2}\right)}\right]
$$

where $b=\sqrt{\frac{1-\mu}{1+\mu}}$. In Fig. 2 (left panel) we plot the response time as a function of the parameter $\mu$ for a given value of the perturbation amplitude $p_{0}$. Note that below the excitability threshold, $p_{0}<2 \arccos (\mu)$ (equivalently $\mu<\cos \left(p_{0} / 2\right)$ ), $t_{r}$ does not exist. For $\mu>\cos \left(p_{0} / 2\right)$ the response time $t_{r}$ is a decreasing function of $\mu$ which approaches zero as $\mu \rightarrow 1$. This result shows that the response time of an Adler system to an above-threshold external perturbation progressively decreases as the Andronov bifurcation point $(|\mu|=1)$ is approached, in agreement with the numerical result shown in Fig. 2 (right panel).
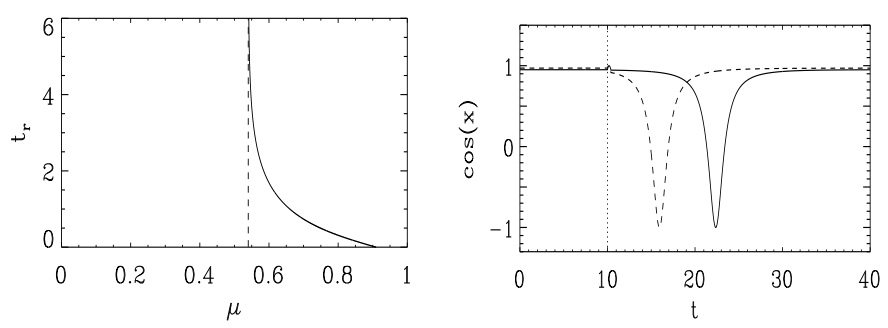

FIG. 2: Left panel: Response time $t_{r}$ versus $\mu$ for the Adler system $x$ perturbed by $p_{0} \delta(t)$ with $p_{0}=2$ from Eq. 6 Right panel: time series for $x(t)$ for $\mu=0.95$ (solid line) and $\mu=0.97$ (dashed line). Both systems have been perturbed at $t_{0}=10$ by a pulse of constant amplitude $p=1.7$ and duration $\Delta t=0.4$. Note that, in agreement with the left panel, the system with the larger value of $\mu$ pulses before the one with the smaller value.

The fact that the response time decreases with lower excitability threshold, and that in the coupled system the slave can emit pulses that are not followed by a pulse in the master, suggest that the mechanism for anticipation in the masterslave configuration is that the slave has a lower excitability threshold than the master. This is supported by the following qualitative argument: Imagine that at $t=t_{0}$ both systems, master and slave, are in the rest state $x\left(t_{0}^{-}\right)=y\left(t_{0}^{-}\right)=x_{-}$. The effect of the perturbation changes both values to $x\left(t_{0}^{+}\right)=$ $x\left(t_{0}^{-}\right)+p_{0}, y\left(t_{0}^{+}\right)=y\left(t_{0}^{-}\right)+p_{0}$. Due to the coupling, the slave can be considered to have at this time an effective $\mu_{\mathrm{eff}}\left(t_{0}\right)=\mu+K\left[x\left(t_{0}^{+}\right)-y\left(t_{0}^{+}-\tau\right)\right]=\mu+K p_{0}$. Since $\mu_{\text {eff }}(t)>\mu$ also for all times $t$ such that $t_{0} \leq t<t_{0}+\tau$, the excitability threshold of the slave has been reduced and the response time decreases.

To give a more rigorous evidence for this explanation, we consider now two coupled systems, Eqs. 45, in the presence of a single perturbation which we choose to be a pulse of constant amplitude $p$ and duration $\Delta t$ acting at time $t_{0}$ in which both systems are in the rest state $x\left(t_{0}^{-}\right)=y\left(t_{0}^{-}\right)=x_{-}$. The results are reported in fig 3

For a sufficiently large perturbation, the master and the slave respond with an excitable spike and the slave pulse anticipates the master pulse (fig. 3a). For small perturbation amplitude no pulses are generated and both systems respond proportionally to the applied stimulus (fig 4c). However, an intermediate amplitude of the perturbation triggers the emission of an excitable pulse by the slave system while the master responds linearly (fig $3 b$ ). This confirms a lowering of the 


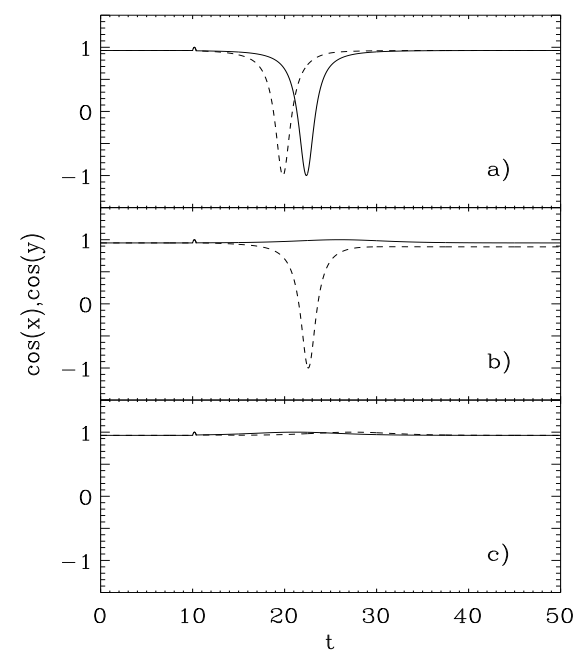

FIG. 3: Response of the master (solid line) and slave (dashed line) for three different amplitudes of the singular perturbation of duration $\Delta t=0.4$ at time $t_{0}=10$ : (a) $p=1.7$, (b) $p=1.65$ and (c) $p=1.61$. Other parameters are $\mu=0.95, \tau=5$ and $K=0.01$.

excitability threshold of the slave as compared to the master, which is systematically found for all coupling parameters that yield anticipating synchronization. In Fig. 4 4 we plot the ratio $R$ between the minimum amplitude of the perturbation which generates an excitable pulse in the slave and the minimum amplitude that generates a pulse in the master. As shown in the figures, the effect of this particular coupling scheme on the slave system is to lower its excitability threshold in such way that the difference between the response time of the master and the slave to an external perturbation equals approximately the delay in the coupling term, $\tau$. It is worth noting that when $K$ or $\tau$ tend to zero, not surprisingly the thresholds for the slave and the master tend to be equal, while for large values of $\tau$ the difference between the two thresholds is very large.

Clearly, the same reasoning can be followed if the perturbation applied to both systems is a white noise process. This allows us to explain why the erroneous synchronization events correspond to the slave system firing a pulse that is not followed by a pulse in the master: for a particular noise level the master response is proportional to the perturbation while the slave emits an excitable pulse. By increasing the noise level both master and slave emit excitable pulses, each pulse of the slave being anticipated respect to that of the master.

Since, as we have shown, master and slave systems respond to external perturbations with different response times, a question which arises is whether it is possible to chose the parameters such that the anticipation time is arbitrarily large, in particular, larger than the master response time, $\tau>t_{r}$, a result that would violate the causality principle. In order to answer this question, we plot in Fig. (5) the results of integrating Eqs. 45 under the effects of a single perturbation for three different values of the parameter $\tau$. When $\tau<t_{r}(5 \mathrm{~h})$ or $\tau \approx t_{r}(5 \mathrm{~b})$,

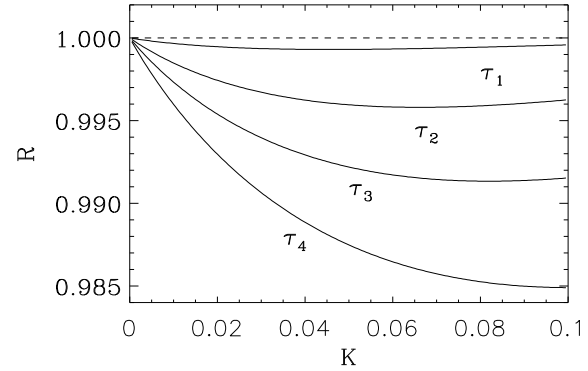

FIG. 4: The ratio between the slave and the master excitability threshold as a function of $K$ for $\tau_{1}=0.05, \tau_{2}=0.2, \tau_{3}=0.35$ and $\tau_{4}=0.5$. Considered system have parameter $\mu=0.95$. Perturbation is applied at time $t_{0}=10$ with magnitude $p=1.635$ and duration $\Delta t=0.4$. The dashed line corresponds to the constant excitability threshold of the master.

the anticipation time is approximately equal to $\tau$. However, when $\tau \gg t_{r}$, the anticipation time greatly differs from the delay time, such that the slave anticipates the master by a time interval always lower than $t_{r}$ (55). This is a reasonable limit to the anticipation time: the pulse cannot anticipate the perturbation which created it. In other words, master and slave are both "slaves" of the external perturbation, although the presence of the master signal into the coupling term contributes to lower the excitability threshold of the slave leading to the anticipation phenomenon.

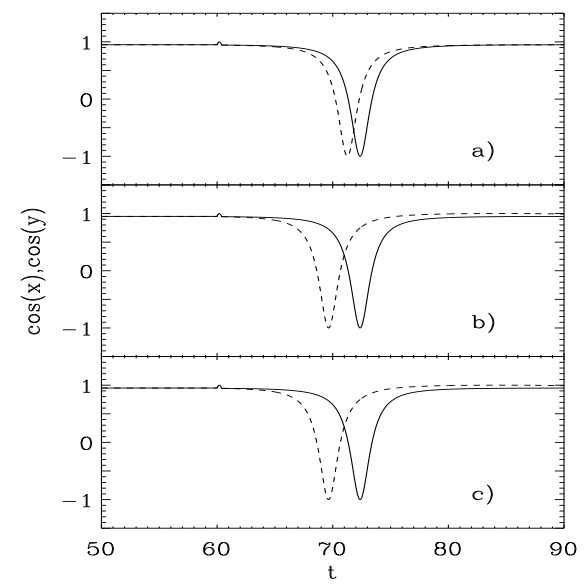

FIG. 5: Two coupled systems (master and slave) with a coupling parameter $K=0.01$ and delay time (a) $\tau=1$, (b) $\tau=5$ and (c) $\tau=50$. Both systems have $\mu=0.95$ and are perturbed at time $t_{0}=60$ with a pulse of magnitude $p=1.7$ and duration $\Delta t=0.4$.

In order to assess the generality of our hypothesis, we have also considered two delayed coupled FitzHugh-Nagumo sys- 
tems:

$$
\begin{aligned}
& \left(\dot{x_{1}}, \dot{x_{2}}\right)=\left(x_{2}+x_{1}-\frac{x_{1}^{3}}{3}, \epsilon\left(a-x_{1}\right)\right) \\
& \left(\dot{y_{1}}, \dot{y_{2}}\right)=\left(y_{2}+y_{1}-\frac{y_{1}^{3}}{3}+K\left(x_{1}-y_{1}^{\tau}\right), \epsilon\left(a-y_{1}\right)\right)
\end{aligned}
$$

In the excitable regime, which occurs when $|a|>1$, the system possesses a single steady state. As the critical value $\left|a_{c}\right|=1$ is approached, the excitability threshold is lowered [15]. In this sense, the control parameter $a$ plays the same role as the parameter $\mu$ in Adler's equation. In fact, we have checked that also in this case the response time of the system to an external perturbation decreases as the critical value $a_{c}$ is approached (see Fig. 6.

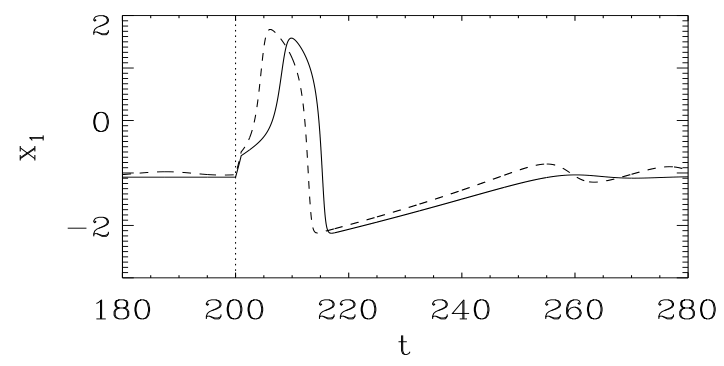

FIG. 6: Time series for the variable $x_{1}$ of the FitzHugh-Nagumo system for $a=1.01$ (dashed line) and $a=1.08$ (solid line). In both cases it is $\epsilon=0.09$. As indicated by the vertical dotted line, the system is perturbed at a time $t_{0}=200$ by a pulse of amplitude $p=0.4$ and duration $\Delta t=1$. Note that the response time decreases with increasing $a$.

We now consider the unidirectionally delayed coupled system. We find, as in the Adler's system, that the excitability threshold for the slave is lower than that of the master, as shown in Fig. 7 and that the maximum anticipation time is limited by the response time of the master. Finally, we note that we have also found exactly the same phenomenology for two delayed coupled Hodgkin-Huxley systems.

The ubiquity of this effect is, in our opinion, an indication that the lowering of the excitability threshold of the slave in a delayed coupling scheme is a general mechanism for anticipating synchronization in excitable systems. This mechanism allows to explain all the observations in the regime of anticipating synchronization, in particular the erroneous firing of pulses in the slave system. In addition, it evidences the causality of this phenomenon: the master and slave systems follow the applied external perturbations, although the response time of the slave system is shorter due to the effects of the coupling. Moreover, we have shown that the anticipation time is limited by the response time of the master system. The relevance of this type of mechanism for the synchronization of coupled chaotic systems is an open question that will be studied in the near future.

We acknowledge financial support from MCYT (Spain) and

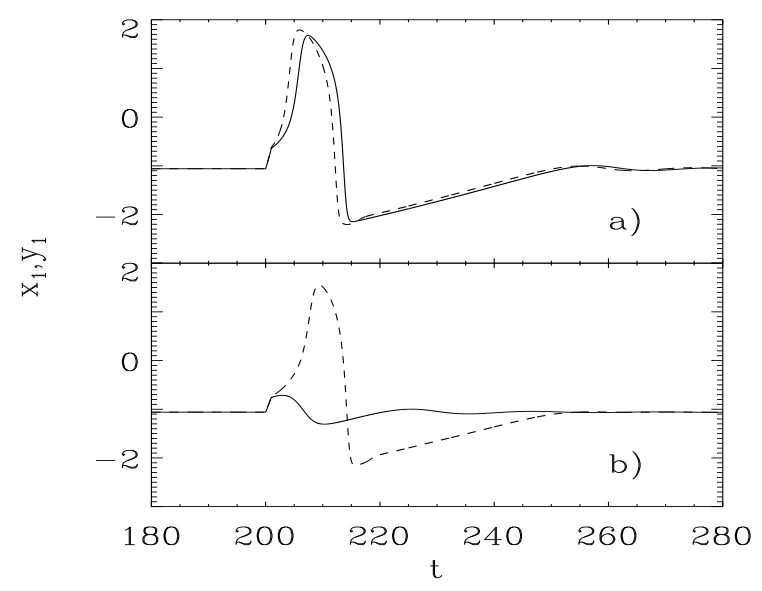

FIG. 7: Response of the master ( $x_{1}$, solid line) and slave ( $y_{1}$, dashed line) for two coupled FitzHugh-Nagumo systems with $a=1.01, \epsilon=$ $0.09, \tau=4, K=0.1$, after perturbation at $t_{0}=200$ by a pulse of amplitude $p$ and duration $\Delta t=1$. For large amplitude, $p=0.4$, case (a), both systems pulse whereas for the smaller amplitude, $p=0.3$, there is only pulse in the slave variable.

FEDER through projects TIC2002-04255-C04, BFM20001108 and BFM2001-0341-C02-01. S.B. acknowledges financial support from MEC through sabbatical grant PR20020329.

[1] A. Pikovsky, M. Rosemblum and J. Kurths, Synchronization: A universal concept in nonlinear sciences, Cambridge University Press (2001).

[2] L. Pecora, T. Carrol, G. Johnson and D. Mar, Chaos 7520 (1997).

[3] H. U. Voss, Phys. Rev. E 61, 5115 (2000); Phys. Rev. E 64, 039904(E) (2001); Phys. Rev. Lett. 87, 014102 (2001).

[4] E. Hernandez-Garcia, C. Masoller, C.R. Mirasso Phys. Lett. A 29539 (2002)

[5] O. Calvo, D.R. Chialvo, V.M. Eguiluz, C.R. Mirasso and R. Toral, Chaos 147 (2003)

[6] C. Masoller, Phys. Rev. Lett. 862782 (2001).

[7] H. U. Voss, Int. J. of Bifurc. and Chaos 121619 (2002)

[8] Y. Liu, Y. Takiguchi, P. Davis. T. Aida, S. Saito and J.M. Liu, Appl. Phys. Lett. 804306 (2002)

[9] M. Ciszak, O. Calvo, C. Masoller, C. Mirasso, R. Toral, Phys. Rev. Lett. 90 (204102) (2003); R. Toral, C. Masoller, C. Mirasso, M. Ciszak and O. Calvo Physica A 325, 192 (2003).

[10] R. FitzHugh, Biophys. J.,1, 445 (1961); J. Nagumo, S. Arimoto, S. Yoshizawa, Proc. IREE aust, 50, 2061 (1962).

[11] A.Hodgkin, A. Huxley, J. Physil.(Lond.), 117, 500-544.

[12] M. Eguia, G. Mindlin Phys. Rev. E 61, 6490 (2000).

[13] P. Coullet, T. Frisch, J.M. Gilli, S. Rica, Chaos 4, 485 (1994).

[14] A.A. Andronov, E.A. Leontovich, J.J. Gordon, A.G. Maier, "Theory of bifurcations of dynamic systems on a plane" (Wiley, New York 1973).

[15] S. Barland, O. Piro, M. Giudici, J. Tredicce, S. Balle, Phys. Rev. E, 68, 036209 (2003). 